\title{
THE MAXIMAL ABELIAN SUB-EXTENSION DETERMINES WEAK MIXING FOR GROUP EXTENSIONS
}

\author{
E. ARTHUR ROBINSON, JR.
}

(Communicated by R. Daniel Mauldin)

\begin{abstract}
Weak mixing is an important property for group extensions because its absence is the principal obstruction to lifting a large number of stronger mixing properties. Whether or not an extension is weakly mixing can be determined by studying the sub-extension corresponding to the quotient by the commutator subgroup. Several conditions equivalent to weak mixing are given. In particular, an extension by any group with no abelian factors (for example any nonabelian simple group) is automatically weak mixing if it is ergodic. The proof uses spectral multiplicity theory.
\end{abstract}

\section{INTRODUCTION}

In $[\mathrm{KN}]$ Keynes and Newton showed that a compact affine extension is ergodic if and only if the maximal equicontinuous sub-extension is ergodic. Similarly, Zimmer [Z2] showed that a compact nilmanifold extension is ergodic if and only its maximal toral sub-extension is ergodic. Both of these results say that some ergodic property holds for an extension once it holds for a certain sub-extension. In this paper we prove another result of the same type. We show that an ergodic compact group extension is weakly mixing if and only if its maximal abelian sub-extension is weakly mixing. This reduces the question of weak mixing for group extensions to the case of abelian group extensions, where necessary and sufficient conditions are already well known (cf. [JP]). Conditions for weak mixing are important because weak mixing is the key hypothesis for a large number of theorems which state that if $T$ has a certain strong mixing property and $\widetilde{T}$ is a weakly mixing group extension of $T$, then $\widetilde{T}$ also has the strong mixing property. Such theorems are known as lifting theorems, and hold, for example, for mixing, multiple mixing [Rul], the K-property [JP], and the Bernoulli property [Ru2].

Let $T$ be an ergodic measure preserving transformation on a Lebesgue probability space $(X, \mu)$. Let $G$ be a compact metric group with Haar measure $\gamma$. Let $\Phi(X, G)$ denote the set of all measurable maps $\phi: X \rightarrow G$. For $\phi \in \Phi(X, G)$ we consider the compact group extension, or $G$ extension $\widetilde{T}$

Received by the editors July 29, 1990.

1980 Mathematics Subject Classification (1985 Revision). Primary 28D05, 28D20.

Partially supported by NSF DMS 8805340 . 
of $T$

$$
\widetilde{T}(x, g)=(T x, \phi(x) g),
$$

where $(x, g) \in(\tilde{X}, \widetilde{\mu})=(X \times G, \mu \times \gamma)$. The function $\phi$ is called the cocycle for the extension. When $G$ is abelian we call $\widetilde{T}$ an abelian extension. It is well known that an extension $\widetilde{T}$ is ergodic if and only if $T$ is ergodic and the essential range of $\phi$ is $G$ (cf. [Z1]). Another characterization of ergodicity in the abelian case is given in Lemma 1 below. An obvious necessary condition for weak mixing in $\widetilde{T}$ is for $T$ to be weakly mixing. A transformation fails to be weakly mixing if and only if it has a nontrivial factor with discrete spectrum, and there is a unique maximal factor of this type. We say that $\widetilde{T}$ is a continuous extension of $T$ if $\widetilde{T}$ and $T$ have the same maximal discrete spectrum factor. An extension $\widetilde{T}$ is weakly mixing if and only if it is a continuous extension of a weakly mixing transformation $T$.

\section{The MAXIMAL ABELIAN SUB-EXTENSION}

Given a $G$ extension $\widetilde{T}$ of $T$, let $\mathscr{R}_{h}, h \in G$, denote the natural right action of $G$ on $\tilde{X}, \mathscr{R}_{h}(x, g)=\left(x, g h^{-1}\right)$. If $K$ is a closed normal subgroup of $G$, then $K$ acts on $\widetilde{X}$ by restricting $\mathscr{R}_{h}$ to $K$. Let $\left(\widetilde{X}_{K}, \tilde{\mu}_{K}\right)=\left(X \times G / K, \mu \times \gamma_{G / K}\right)$ denote the factor of $(\tilde{X}, \tilde{\mu}) \bmod$ the orbits of $K$, where $\gamma_{G / K}$ is Haar measure on $G / K$. Let $\widetilde{T}_{K}$ denote the induced factor transformation

$$
\widetilde{T}_{K}(x, g K)=(T x, \phi(x) g K)=\left(T x, \phi_{K}(x) g K\right),
$$

where $(x, g K) \in\left(\tilde{X}_{K}, \tilde{\mu}_{K}\right), \pi_{K}: G \rightarrow G / K$ is the canonical projection, and $\phi_{K} \in \Phi(X, G / K)$ is defined $\phi_{K}(x)=\pi_{K}(\phi(x))$. We call $\widetilde{T}_{K}$ a sub-extension of $\widetilde{T}$. By (2), $\widetilde{T}_{K}$ is a $G / K$ extension of $T$, and since $\widetilde{T}$ commutes with a free $K$ action (cf. [R2]), $\widetilde{T}$ is a $K$ extension of $\widetilde{T}_{K}$.

If $[G, G] \subseteq G$ is the commutator subgroup then $G /[G, G]$ is an abelian group called the maximal abelian factor of $G$. We denote $\widetilde{T}_{[G, G]}$ by $\widehat{T}$ and observe that $\widehat{T}$ is an abelian group extension of $T$. We call $\widehat{T}$ the maximal abelian sub-extension of the extension $\widetilde{T}$.

Theorem 1. Let $G$ be a compact metric group. Suppose the $G$ extension $\tilde{T}$ of $T$ is ergodic. Then $\widetilde{T}$ is a continuous extension of $\widehat{T}$. In particular, $\widetilde{T}$ is a continuous extension of $T$ if and only if $\widehat{T}$ is a continuous extension of $T$.

Proof. Let $L_{g}$ denote the left-regular representation of $G$ on $L^{2}(G, \gamma)$. By the Peter-Weyl theorem there exists an orthogonal decomposition

$$
L^{2}(G, \gamma)=\bigoplus_{j=1}^{t} \bigoplus_{k=1}^{d_{j}} H_{j, k},
$$

$t \leq \infty$, such that each $H_{j, k}$ is $L_{g}$ invariant, and the restriction of $L_{g}$ to $H_{j, k}$, denoted $L_{g}^{j, k}$, is irreducible. For each $j$ and $k$ the $\operatorname{dim}\left(H_{j, k}\right)=d_{j}$. Also $L_{g}^{j, k}$ and $L_{g}^{j, k^{\prime}}$ are equivalent. Let $P_{j, k}$ denote the projection to $H_{j, k}$. 
Let $U_{\widetilde{T}}$ be the induced unitary operator for $\widetilde{T}$ on $L^{2}(\widetilde{X}, \widetilde{\mu})$, defined $U_{\widetilde{T}} f(\tilde{x})$ $=f(T \tilde{x})$. There is natural orthogonal decomposition of $L^{2}(\widetilde{X}, \widetilde{\mu})$ into $U_{\widetilde{T}}$ invariant subspaces corresponding to (3) (cf. [R1]),

$$
L^{2}(\tilde{X}, \tilde{\mu})=\bigoplus_{j=1}^{t} \bigoplus_{k=1}^{d_{j}} \mathscr{H}_{j, k} .
$$

In particular, the projection $\mathscr{P}_{j, k}$ to $\mathscr{H}_{j, k}$ is simply $P_{j, k}$ applied to $f(x, g)$ viewed as a function of $g \in G$ for $\mu$ a.e. $x \in X$. The decomposition (4) has the property that for any $j$, the restriction of $U_{\widetilde{T}}$ to $\mathscr{H}_{j, k}$ and to $\mathscr{H}_{j, k^{\prime}}$ are unitarily equivalent [R1]. We implement this equivalence via a unitary operator $W$, commuting with $U_{\widetilde{T}}$, such that for all $j$ and $k, W\left(\mathscr{H}_{j, k}\right)=\mathscr{H}_{j, k^{\prime}}$, where $k^{\prime}=k+1$ if $k<d_{j}$ and $k^{\prime}=1$ if $k=d_{j}$. When $d_{j}=1$ we assume $W$ is the identity. Notice that $W$ is not the identity if $d_{j} \neq 1$. Such a $d_{j}$ always exists when $G$ is nonabelian [R1], and we may assume with no loss of generality that $G$ is nonabelian, since otherwise $[G, G]$ is trivial and $\widehat{T}=\widetilde{T}$.

In (3), $d_{j}=1$ when $\operatorname{dim}\left(L_{g}^{j, 1}\right)=1$. Each 1-dimensional representation is associated with a factor of $G$ isomorphic to the circle, namely $G / \operatorname{ker}\left(L_{g}^{j, 1}\right)$. For a closed normal subgroup $K$ of $G, G / K$ is abelian if and only if $[G, G] \subseteq$ $K$, and if $G / K$ is abelian then there exists a closed subgroup $J \subseteq G /[G, G]$ with $G / K$ isomorphic to $(G /[G, G]) / J$. Thus the 1-dimensional representations of $G$ correspond to the irreducible representations of $G /[G, G]$. We may assume in (3) that $H_{1,1}$ corresponds to the trivial representation, and that there exists $t_{0} \geq 1$ such that $d_{j}=1$ for all $j \leq t_{0}$ and $d_{j}>1$ for $t_{0}<j \leq t$. Then the decomposition (4) for $U_{\widehat{T}}$ looks like

$$
L^{2}(\widehat{X}, \widehat{\mu})=\bigoplus_{j=1}^{t_{0}} \bigoplus_{k=1}^{d_{j}} \mathscr{H}_{j, k} .
$$

Moreover, we can identify $L^{2}(X, \mu)$ with $\mathscr{H}_{1,1}$.

Now suppose $f$ is an eigenfunction for $\widetilde{T}$ (and thus for $U_{\widetilde{T}}$ ) with eigenvalue $\lambda$. Then

$$
U_{\widetilde{T}}\left(\mathscr{P}_{j, k} f\right)(\tilde{x})=\mathscr{P}_{j, k} U_{\widetilde{T}} f(\tilde{x})=\mathscr{P}_{j, k}(f \circ \tilde{T})(\tilde{x})=\mathscr{P}_{j, k}(\lambda f)(\tilde{x})=\lambda\left(\mathscr{P}_{j, k} f\right)(\tilde{x}),
$$

so that $\mathscr{P}_{j, k} f$ is either itself an eigenfunction with eigenvalue $\lambda$, or $\mathscr{P}_{j, k} f=0$. If $\mathscr{P}_{j, k} f=0$ for all $j>t_{0}$, then $f$ is an eigenfunction for $\widehat{T}$. Thus we suppose that $\mathscr{P}_{j, k} f \neq 0$ for some $j>t_{0}$ and some $k$. Then $\mathscr{P}_{j, k} f$ and $W \mathscr{P}_{j, k} f$ are both eigenfunctions with eigenvalue $\lambda$, and $\mathscr{P}_{j, k} f \perp W \mathscr{P}_{j, k} f$ since $\mathscr{P}_{j, k} f \in \mathscr{H}_{j, k}$ and $W \mathscr{P}_{j, k} f=\mathscr{P}_{j, k^{\prime}} f \in \mathscr{H}_{j, k^{\prime}}$. This implies that the multiplicity of $\lambda$ is greater than one, contradicting the ergodicity of $\widetilde{T}$.

\section{NECESSARY AND SUFFICIENT CONDITIONS FOR WEAK MIXING}

Let $\phi, \phi^{\prime} \in \Phi(X, G)$ and let $K$ be a closed normal subgroup. We say $\phi$ and $\phi^{\prime}$ are cohomologous $\bmod K$ if

$$
\psi(T x) \phi_{K}(x)=\phi_{K}^{\prime}(x) \psi(x),
$$


for some $\psi \in \Phi(X, G / K)$. If $K=\{e\}$, then we say that $\phi$ and $\phi^{\prime}$ are cohomologous. If $\phi$ and $\phi^{\prime}$ are cohomologous mod $K$ then the corresponding sub-extensions $\widetilde{T}_{K}$ and $\widetilde{T}_{K}^{\prime}$ are isomorphic. In particular, letting $Z(x, g)=$ $(x, \psi(x) g)$, we have

$$
Z \circ \widetilde{T}_{K}=\tilde{T}_{K}^{\prime} \circ Z
$$

If there exists $h \in G / K$ such that

$$
\psi(T x) \phi_{K}(x)=h \psi(x)
$$

then $\phi$ is cohomologous to the constant $h \bmod K$. We refer to such a $\phi$ as an almost coboundary mod $K$. If $h=e, \phi$ is called a coboundary mod $K$. If $[G, G] \subseteq K$ then (8) is equivalent to

$$
\phi_{K}(x) h^{-1}=\psi^{-1}(T x) \psi(x) .
$$

For $1 \leq j \leq t_{0}$, we have $d_{j}=1$, and we let $\chi_{j}(g)=L_{g}^{j, 1}$. These are the 1-dimensional representations of $G$. We let $\Gamma_{G}$ denote the set of all $\chi_{j}$. If $G$ is abelian then $\Gamma_{G}$ is the dual group of $G$ under pointwise multiplication. If for some $\chi \in \Gamma_{G}$ there exists a complex valued measurable function $\psi$ and a complex number $|\lambda|=1$ such that

$$
(\chi \circ \phi)(x) \lambda^{-1}=\psi^{-1}(T x) \psi(x)
$$

then we say $\phi$ is cohomologous to the constant $\lambda \bmod \chi$ and we call $\phi$ an almost-coboundary mod $\chi$. When $\lambda=1, \phi$ is called a coboundary mod $\chi$. For $\chi \in \Gamma_{G}$, if we let $K=\operatorname{ker}(\chi)$, then $\pi_{K}=\chi$ and (8) is equivalent to (10) with $\lambda=\chi(h)$.

Theorem 2. Let $T$ be weakly mixing and let $\tilde{T}$ be an ergodic extension of $T$ by $G$ with $\phi \in \Phi(X, G)$. Let $\widehat{T}$ be the maximal abelian factor extension of $\widetilde{T}$. Then the following are equivalent:

(i) $\widetilde{T}$ is weakly mixing.

(ii) $\widetilde{T}$ is a continuous extension of $T$.

(iii) $\widehat{T}$ is a continuous extension of $T$.

(iv) $\widehat{T}$ is weakly mixing.

(v) For all $\chi \in \Gamma_{G}, \phi$ is not an almost coboundary mod $\chi$.

(vi) For each $h \in G /[G, G], \mathscr{R}_{h} \circ \widehat{T}$ is ergodic.

(vii) For any closed normal subgroup $K$ of $G, \phi$ is not an almost coboundary $\bmod K$.

The proof of Theorem 2 depends on the following results about abelian extensions [JP].

Lemma 1. Let $G$ be abelian and suppose $\tilde{T}$ is a $G$ extension of $T$.

(1) If $T$ is ergodic then $\tilde{T}$ is ergodic if and only if $\phi$ is not a coboundary $\bmod$ any $\chi \in \Gamma_{G}$.

(2) If $T$ is ergodic, then $\lambda$ is an eigenvalue of $\tilde{T}$ which is not an eigenvalue of $T$ if and only if $\phi$ is cohomologous to $\lambda \bmod \chi$ for some $\chi \in \Gamma_{G}$.

(3) In particular, if $T$ is weakly mixing then $\tilde{T}$ is weakly mixing if and only if $\phi$ is not an almost coboundary mod any $\chi \in \Gamma_{G}$. 
Proof of Theorem 2. It follows from Theorem 1 that (i)-(iv) are equivalent.

For any $h \in G /[G, G], \mathscr{R}_{h} \circ \widehat{T}$ is an abelian extension: $\left(\mathscr{R}_{h} \circ \widetilde{T}\right)(x, g)=$ $\left(T x, \phi(x) h^{-1} g\right)$. It follows from Lemma 1 that $\mathscr{R}_{h} \circ \widetilde{T}$ is ergodic if and only if $\phi(x) \cdot h^{-1}$ is not a coboundary mod any $\chi \in \Gamma_{G /[G, G]}$, or equivalently, $\phi$ is not an almost coboundary mod $\chi$. Since we can identify $\Gamma_{G}=\Gamma_{G /[G, G]}$, (v) and (vi) are equivalent.

Now assume (v) does not hold. Then $\phi$ satisfies (10) for some $\lambda$ and $\psi$, so that by Lemma $1, \widehat{T}$ is not weakly mixing. Conversely, if $\widehat{T}$ is not weakly mixing then (9) holds by Lemma 1 , contradicting (v).

For $\chi \in \Gamma_{G}$, we have that not (v) implies not (vii) since (9) and (10) are equivalent for $K=\operatorname{Ker}(\chi)$ and $\lambda=\chi(h)$. Conversely, assume not (vii) and let $K$ be such that (8) holds. Since (8) is a special case of (6), we have (7), so that $\widetilde{T}_{K}$ is isomorphic $T \times \Theta_{h}$, where $\Theta_{h}$ is the left rotation by $h$ on $G / K$. It follows that $\Theta_{h}$ is a factor of $\widetilde{T}_{K}$. Since $\widetilde{T}$ is ergodic, $\Theta_{h}$ is ergodic, and thus uniquely ergodic, so that the subgroup $F=\left\{h^{n}: n \in \mathbf{Z}\right\}$ is dense in $G / K$. Since $F$ is abelian, $G / K$ must be abelian, and so $[G, G] \subseteq K$. Let $J$ be the closed subgroup of $G /[G, G]$ such that $(G /[G, G]) / J$ is isomorphic to $G / K$. Then $\chi \in \Gamma_{G /[G, G]}$ vanishes on $K$ if and only if it vanishes on $J$. Thus there exists $\chi$ such that $K \subseteq \operatorname{ker}(\chi)$. It follows that $\phi$ is an almost coboundary mod $\chi$, which implies not $(\mathrm{v})$.

Let $G$ be a group such that for any closed normal subgroup $K, G / K$ is nonabelian. Equivalently $[G, G]=G$. We say $G$ has no abelian factors. In particular, any simple nonabelian group $G$ (e.g. $A_{5}$ ) has no abelian factors.

Corollary 1. Suppose $G$ has no abelian factors. Let $\widetilde{T}$ be a $G$ extension of $T$ and suppose $T$ is weakly mixing. If $\widetilde{T}$ is ergodic then $\widetilde{T}$ is weakly mixing.

For example, since the Bernoulli property satisfies a lifting theorem (cf. $\S 1$ ), Corollary 1 shows that any ergodic $A_{5}$ extension $\widetilde{T}$ of a Bernoulli shift must be Bernoulli.

Corollary 2. Let $T$ be ergodic and let $\widetilde{T}$ be a $G$ extension of $T$. Then $\widetilde{T}$ is a continuous extension of $T$ if and only if the cocycle $\phi$ is not cohomologous to a constant mod any $\chi \in \Gamma_{G}$.

Comment 1. Suppose $T$ is weakly mixing and that $\phi$ is a an almost coboundary $\bmod K_{\chi}$ for all $\chi \in \Gamma_{G}$. It is easy to see in this case that $\phi$ is an almost coboundary since $\widetilde{T}$ is isomorphic to a product of $T$ and a rotation. However, if $T$ is not weakly mixing the situation can be completely different, as the following example shows. Let $T$ be the rotation on the circle by $4 \pi \alpha$, where $\alpha$ is irrational, and let $\widetilde{T}$ be rotation by $2 \pi \alpha$. Then $\widetilde{T}$ is isomorphic to a $Z / 2$ extension of $T$, and $\widetilde{T}$ has new eigenvalues $\lambda^{n}=e^{2 \pi i n \alpha}$ for $n$ odd. By part (2) of Lemma $1, \phi$ is an almost coboundary mod $\chi(k)=(-1)^{k}$. However, $\phi$ is not cohomologous to any constant. Indeed, the only possible constants are 1 or -1 . But $\phi$ is not cohomologous to 1 since $\widetilde{T}$ is ergodic, and $\phi$ is not cohomologous to -1 , since that would imply $\lambda=-1$ is an eigenvalue, contradicting the irrationality of $\alpha$.

Comment 2. Results similar to Theorems 1 and 2 do not hold for ergodicity: Let $X=\{x\}$ and let $T$ be the identity on $X$. Any $G$ extension $\widetilde{T}$ of $T$, with 
$\mathrm{G}$ nonabelian, is just a rotation on $G$ by some $h \in G$. Since $G$ is not abelian, $\widetilde{T}$ is not ergodic. However, by a careful choice of $G$ and $h$, the transformation $\widehat{T}$, which is a rotation on $G /[G, G]$, can be made to be ergodic.

\section{GENERICITY}

Given a compact metric group $G$, let us fix an invariant metric $d_{G}$ satisfying $d_{G}(g, h) \leq 1$ for all $g, h \in G$. We define a metric $d$ on $\Phi(X, G)$ by

$$
d\left(\phi, \phi^{\prime}\right)=\int_{X} d_{G}\left(\phi(x), \phi^{\prime}(x)\right) d \mu
$$

with pointwise multiplication $\Phi(X, G)$ is a metric group with invariant metric $d$. It is easy to see that $d$ gives the topology of convergence in measure. Let $G^{\prime}=G /[G, G]$. In the same manner as above, we define an invariant metric $d^{\prime}$ on $\Phi\left(X, G^{\prime}\right)$ based on a bounded invariant metric $d_{G^{\prime}}$ for $G^{\prime}$.

Let us fix a measure preserving transformation $T$. We denote the extension (1) corresponding to a particular $\phi \in \Phi(X, G)$ by $\tilde{T}_{\phi}$. We will identify properties of the extensions $\widetilde{T}_{\phi}$ with subsets $\Phi_{0} \subseteq \Phi(X, G)$, namely, $\Phi_{0}$ is the set of all $\phi$ such that $\tilde{T}_{\phi}$ has the given property. We say that a given property is generic for $G$ extensions $\widetilde{T}$ of $T$ if the set $\Phi_{0}$ corresponding to that property contains a dense $G_{\delta}$ subset of $\Phi(X, G)$. The following result is well known (for the abelian case, see [JP]).

Proposition 1. Suppose $T$ is ergodic and aperiodic. Then there is a dense $G_{\delta}$ subset $\Phi_{\mathscr{C}}$ of $\Phi(X, G)$ so that for $\phi \in \Phi_{\mathscr{E}}$ the extension $\widetilde{T}_{\phi}$ is ergodic. In particular, ergodicity is generic for $G$ extensions $\widetilde{T}$ of an ergodic aperiodic transformation $T$.

Our goal is to prove a similar theorem for weak mixing. The abelian case is proved in [JP].

Theorem 3. Suppose $T$ is weakly mixing. Then there is a dense $G_{\delta}$ subset $\boldsymbol{\Phi}_{\mathscr{W}}$ of $\boldsymbol{\Phi}(X, G)$ so that for $\phi \in \boldsymbol{\Phi}_{\mathscr{W}}$ the extension $\widetilde{T}_{\phi}$ is weakly mixing. In particular, weak mixing is generic for $G$ extensions $\tilde{T}$ of a weakly mixing transformation $T$.

Proof. Consider the mapping $\eta: \Phi(X, G) \rightarrow \Phi\left(X, G^{\prime}\right)$ defined $(\eta \phi)(x)=$ $\pi_{[G, G]}(\phi(x))=\phi_{[G, G]}(x)$. Since the statement of the Theorem 3 is true for abelian groups [JP], there exists a dense $G_{\delta}$ set $\Phi_{\mathscr{E}}^{\prime} \subseteq \Phi\left(X, G^{\prime}\right)$ so that for $\omega \in \Phi_{\mathscr{E}}^{\prime}$ the corresponding $G^{\prime}$ extension $\bar{T}_{\omega}(x, g)=(T x, \omega(x) g)$ is weakly mixing. Since $T$ is weakly mixing it is ergodic and aperiodic. By Proposition 1 there exists a dense $G_{\delta}$ set $\Phi_{\mathscr{E}} \subseteq \Phi(X, G)$ so that for $\phi \in \Phi_{\mathscr{E}}, \widetilde{T}_{\phi}$ is ergodic. Let $\Phi_{1}=\eta^{-1} \Phi_{\mathscr{E}}^{\prime}$. Let $\Phi_{\mathscr{W}}=\Phi_{1} \cap \Phi_{\mathscr{E}}$. Then for $\phi \in \Phi_{\mathscr{W}}$ we have $\widehat{T}_{\phi}=\bar{T}_{\eta \phi}=\bar{T}_{\phi_{[G, G]}}$, and so by Theorem 2 , $\widetilde{T}_{\phi}$ is weakly mixing.

Since $\eta$ is continuous, $\Phi_{1}$ is $G_{\delta}$ in $\Phi(X, G)$. Thus to show that $\Phi_{\mathscr{W}}$ is dense $G_{\delta}$ it is enough to show that $\Phi_{1}$ is dense. To show this it suffices to show that $\eta$ is an open map, for otherwise there exists a ball $B$ in $\Phi(X, G) \backslash \Phi_{1}$ such that $\eta(B)$ contains a ball in $\Phi\left(X, G^{\prime}\right) \backslash \Phi_{\mathscr{E}}^{\prime}$, contradicting the fact that $\Phi_{\mathscr{E}}^{\prime}$ is dense. 
Since $\eta$ is a homomorphism, and since both $\Phi(X, G)$ and $\Phi\left(X, G^{\prime}\right)$ have invariant metrics, it suffices to show that for any neighborhood $B$ of the identity in $\Phi(X, G), \eta(B)$ contains a neighborhood of the identity in $\Phi\left(X, G^{\prime}\right)$. By the open mapping theorem for topological groups (cf. [M]) the canonical homomorphism $\pi_{[G, G]}: G \rightarrow G^{\prime}$ is an open map. Thus for all $\varepsilon_{1}>0$ there exists $\delta_{1}>0$ such that if $d_{G^{\prime}}(h, e)<\delta_{1}$, there exists a $g \in G$ with $d_{G}(g, e)<\varepsilon_{1}$ and $h=\pi_{[G, G]}(g)$.

Let $\varepsilon>0$ be given. Choose $\delta>0$ to satisfy the following two conditions:

(i) $\delta<\varepsilon^{2} / 4$.

(ii) Let $\varepsilon_{1}<\varepsilon / 2$. Choose $\delta_{1}$ for $\varepsilon_{1}$ as above, and let $\delta<\delta_{1}^{2}$.

Now suppose $d(\phi, e)<\delta$ so that $\int_{X} d_{G^{\prime}}\left(\phi_{[G, G]}(x), e\right) d \mu<\delta$. If $E=$ $\left\{x: d_{G^{\prime}}\left(\phi_{[G, G]}(x), e\right)>\sqrt{\delta}\right\}$, then $\mu(E)<\sqrt{\delta}$, and for $x \in X \backslash E$,

$$
d_{G^{\prime}}\left(\phi_{[G, G]}(x), e\right) \leq \sqrt{\delta}<\delta_{1} .
$$

We define $\phi \in \Phi(X, G)$ as follows. In a measurable way, we choose $\phi(x) \in G$ for each $x$ so that

$$
\pi_{[G, G]}(\phi(x))=\phi_{[G, G]}(x),
$$

and using (12) and (ii) so that $\phi(x)$ also satisfies $d_{G^{\prime}}(\phi(x), e)<\varepsilon_{1}$ for $x \in$ $X \backslash E$. We then have

$$
\begin{aligned}
d(\phi, e) & =\int_{X} d_{G}(\phi(x), e) d \mu \\
& =\int_{E} d_{G}(\phi(x), e) d \mu+\int_{X \backslash E} d_{G}(\phi(x), e) d \mu \\
& \leq \int_{E} d \mu+\int_{X \backslash E} \varepsilon_{1} d \mu \leq \sqrt{\delta}+\varepsilon_{1}<\varepsilon / 2+\varepsilon / 2=\varepsilon .
\end{aligned}
$$

It follows from (13) that $\eta \phi=\phi_{[G, G]}$. Thus the $\eta$ image of an $\varepsilon$ ball around $e \in \Phi(X, G)$ contains a $\delta$ ball around $e \in \Phi\left(X, G^{\prime}\right)$, and it follows that $\eta$ is an open map. This implies that $\Phi_{1}$ is dense $G_{\delta}$.

Comment. Using ideas from [JP] together with the proof of Theorem 3, one can show that for $T$ ergodic and aperiodic, the generic $G$ extension $\widetilde{T}$ is a continuous extension.

\section{REFERENCES}

[JP] R. Jones and W. Parry, Compact abelian group extensions of discrete dynamical systems. II, Compositio Math. 25 (1972), 135-147.

[KN] H. B. Keynes and D. Newton, Ergodicity in $(G, \sigma)$ extensions, Global Theory of Dynamical Systems- Proceedings, Northwestern 1979, Lecture Notes in Math., vol. 819, Springer, Berlin.

[M] S. A. Morris, Pontryagin duality and the structure of locally compact abelian groups, London Math Soc. Lecture Note Series, no. 29, Cambridge University Press, Cambridge, England, 1977.

[R1] A. Robinson, Ergodic properties that lift to compact group extensions, Proc. Amer. Math. Soc. 102 (1988), 61-67.

[R2] - Non-abelian extensions have nonsimple spectrum, Compositio Math. 65 (1988), 155-170. 
[Ru1] D. Rudolph, $K$-fold mixing lifts to weakly mixing isometric extensions, Ergodic Theory Dynamical Systems 5 (1985), 445-447.

[Ru2] Classifying the isometric extensions of a Bernoulli shift, J. Analyse Math. 34 (1978), 36-60.

[Z1] R. J. Zimmer, Extensions of ergodic group actions, Illinois J. Math. 20 (1976), 373-409.

[Z2] - Compact nilmanifold extensions of ergodic actions, Trans. Amer. Math. Soc. 223 (1976), 397-406.

Department of Mathematics, The George Washington University, Washington, DC 20052 